\title{
Measuring the non-separability of optical fields
}

Ndagano, B., Sroor, H., McLaren, M., Rosales-Guzmán, C., Forbes, A.

B. Ndagano, H. Sroor, M. McLaren, C. Rosales-Guzmán, A. Forbes, "Measuring the non-separability of optical fields," Proc. SPIE 10120, Complex Light and Optical Forces XI, 101200W (27 February 2017); doi: 10.1117/12.2254028

SPIE. Event: SPIE OPTO, 2017, San Francisco, California, United States 


\title{
Measuring the non-separability of optical fields
}

\author{
Ndagano B. ${ }^{a}$, Sroor H. ${ }^{a}$, McLaren M. $^{a}$, Rosales-Guzmán C. ${ }^{\text {a }}$, and Forbes A. ${ }^{a}$ \\ ${ }^{a}$ School of Physics, University of the Witwatersrand, Private Bag 3, Wits 2050, South Africa
}

\begin{abstract}
Across various areas in the optical world, there has been a growing interest in exploiting the properties of nonseparable optical fields. A class of non-separable fields, known as vector modes, exhibit a coupling between the spatial and polarisation degrees of freedom that is akin of entanglement in quantum mechanics. These vector modes, however, are typically characterized using qualitative measurements which are inadequate in determining to what extent an optical field is non-separable. Here, we present tools to characterize the degree of non-separability of an arbitrary optical field, exploiting the similarities between vector modes and quantum entangled states. As an example, we use vector modes carrying orbital angular momentum to demonstrate the effectiveness of our scheme, and note that the approach can be generalized to vector modes as a whole.
\end{abstract}

Keywords: spatial modes, orbital angular momentum, polarization

\section{INTRODUCTION}

The beam propagation ratio $M^{2}$, is a standard measure of quality that is used to describe laser modes ${ }^{1}$. It is derived from the analogy between probability density functions and the intensity profiles of laser mode $;^{2}$ assuming that intensity profiles can be viewed as the probability of finding the light, the beam width and divergence can be defined represented as second moments o the intensity, leading to the computation of the $M^{2}$. With novel technologies, it is now possible to digitally perform measurements of $M^{2}$. However, this measure is specific to scalar modes; that is, spatial modes of light with uniform polarisation.

Vector modes are a particular class of spatial modes in which the polarization state varies across the profile of the beam ${ }^{3}$. These modes have been of particular interest in our recent classical and quantum studies ${ }^{4-8}$ . They exhibit a non-separable coupling between the spatial and polarization degrees of freedom (DoFs), i.e., the two degrees of freedom cannot be described independently of each other. This definition is reminiscent of a particular type of system in quantum mechanics: entangled particles. Indeed, through entanglement, one or more properties of quantum particles become intertwined. Therefore, it becomes inadequate to describe the entangled particles individually; rather, characterizing the entanglement is essential to the describe the system. Similarly for vector modes, characterizing the degree of non-separability between the DoFs of interest is fundamental to characterizing vector modes. While there has been a plethora of reported methods to generate vector beams ${ }^{9-13}$ , the vector nature of an optical field is commonly deduced from qualitative measurements ${ }^{14,15}$. A quantitative approach is therefore required in determining the degree of 'vectorness'.

Here, we present a scheme to measure the degree of 'vectorness' of an arbitrary vector mode. Similarly to the $M^{2}$ that finds its roots in statistics, we exploit the analogy between the non-separability of vector modes and entanglement in quantum systems, to derive a beam quality measure for vector modes based on quantum tools. By varying the nature of an optical field from purely vector to purely scalar, we show, in comparison with theory, that our scheme allows one to accurately determine the variation in the degree of non-separability.

\section{RESULTS}

Consider a general optical field expressed as follows:

$$
U(\mathbf{r}, \theta)=\cos (\theta) u_{L}(\mathbf{r}) \hat{e}_{L}+\sin (\theta) u_{R}(\mathbf{r}) \hat{e}_{R}
$$

Further author information: (Send correspondence to A.F.)

A.F.: E-mail: andrew.forbes@wits.ac.za, Telephone: +27 117176885

Complex Light and Optical Forces XI, edited by David L. Andrews, Enrique J. Galvez,

Jesper Glückstad, Proc. of SPIE Vol. 10120, 101200W · (C) 2017 SPIE

CCC code: $0277-786 \mathrm{X} / 17 / \$ 18 \cdot$ doi: $10.1117 / 12.2254028$

Proc. of SPIE Vol. $10120101200 \mathrm{~W}-1$ 
(a)

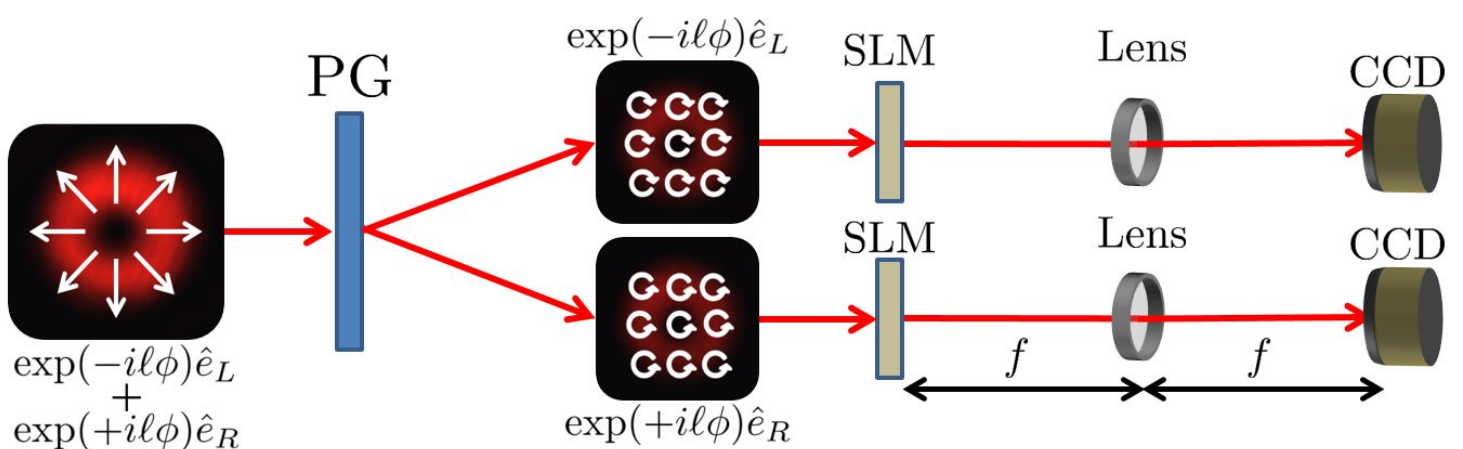

(b)
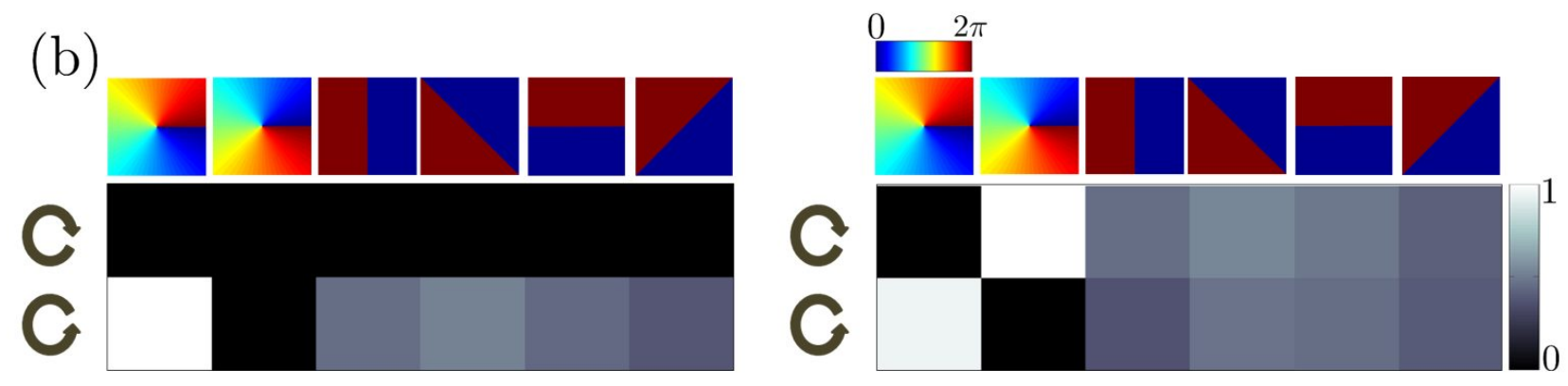

Figure 1. Experimental measurement of the vector quality factor. (a) The left- and right-circular polarization states are spatially separated with a polarization grating (PG) and send onto two SLMs where the OAM eigenstates of the Pauli operators are encoded as digital holograms. The results of optical projections onto the eigenstates are obtained as on-axis intensity measurement in the Fourier plane of a lens placed after the SLMs (b) Shows the results of the projections for a scalar mode (left) and a vector mode(right). The rows represent the two circular polarization states while the columns are the projections onto OAM eigenstates of the Pauli operators. The phase profiles displayed on the top are encoded on the SLMs.

where $\hat{e}_{L}$ and $\hat{e}_{R}$ are the left- and right- circular polarization basis vectors, with associated spatial mode $u_{L}(\mathbf{r})$ and $u_{R}(\mathbf{r})$. By tuning the parameter $\theta$, one changes the nature of the optical field: for $\theta=n \pi / 4$, the field is purely scalar when $n$ is even and purely vector when $n$ is odd. The aim of the scheme is therefore to provide a quantitative measure that will reflect the degree of non-separability of the field in Eq. 1. To this end, we use the concurrence, a quantum measure of entanglement for two-dimensional states. For a non-separable system as in Eq. 1, it is given by $\mathcal{C}=2|\sin (\theta) \cos (\theta)|=|\sin (2 \theta)|$. We define the vector quality factor (VQF) as:

$$
\mathrm{VQF}=\operatorname{Re}\left(\sqrt{1-s^{2}}\right)=|\sin (2 \theta)|
$$

where $s$ is the length of the Bloch vector (also referred to as the degree of polarisation of the averaged polarization state $^{4}$ ), defined as

$$
s^{2}=\sum_{i}\left\langle\sigma_{i}\right\rangle^{2}
$$

The summation index $i=1,2,3$ and the $\left\langle\sigma_{i}\right\rangle$ are the expectation values of the Pauli operators:

$$
\sigma_{1}=\left(\begin{array}{cc}
0 & 1 \\
1 & 0
\end{array}\right) ; \quad \sigma_{2}=\left(\begin{array}{cc}
0 & -i \\
i & 0
\end{array}\right) ; \quad \sigma_{3}=\left(\begin{array}{cc}
1 & 0 \\
0 & -1
\end{array}\right)
$$

The expectation values $\left\langle\sigma_{i}\right\rangle$ are can be obtained from projections onto the eigenstates of each Pauli operator. As an illustrative case, let $U(\mathbf{r}, \theta)$ be a vector vortex modes defined as follows:

$$
U(r, \phi, \theta)=A(r)\left[\cos (\theta) \exp (-i \ell \phi) \hat{e}_{L}+\sin (\theta) \exp (i \ell \phi) \hat{e}_{R}\right]
$$



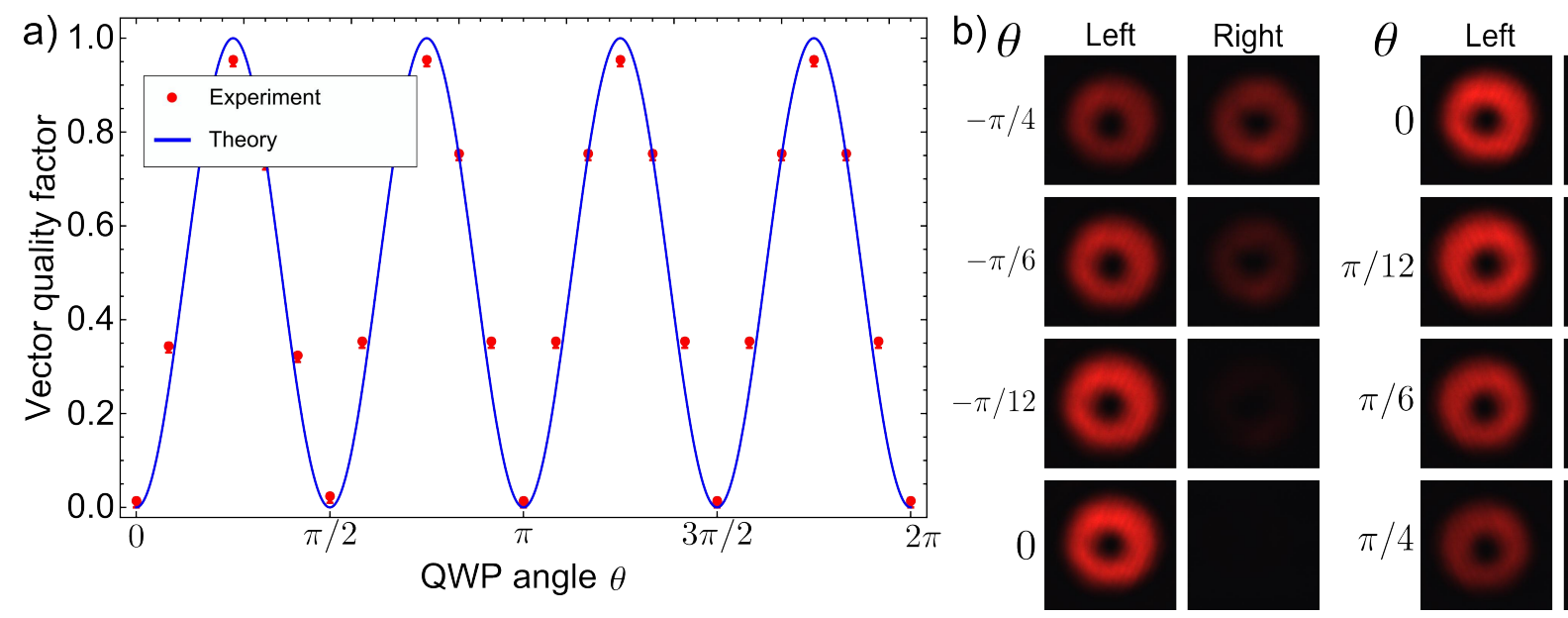

Right

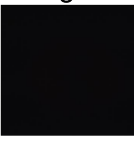

Figure 2. Variation of the vector quality factor as a function of the vectorness of the input mode. The parameter $\theta$ is tuned with a quarter-wave plate, changing the state of polarization before the $q$-plate from linear to circular

where $\ell \in \mathbb{Z}$ is the orbital angular momentum (OAM) index of the mode, with each photon carrying a $\ell \hbar$ quanta of OAM. We generated the vector modes in Eq. 4 using a geometric phase plate called $q$-plate, that transforms as follows

$$
\begin{aligned}
& \exp (i \ell \phi) \hat{e}_{L} \rightarrow \exp [i(\ell+2 q) \phi] \hat{e}_{R}, \\
& \exp (i \ell \phi) \hat{e}_{R} \rightarrow \exp [i(\ell-2 q) \phi] \hat{e}_{L},
\end{aligned}
$$

where $q$ is the charge of the $q$-plate. Thus, a Gaussian mode $(\ell=0)$ with arbitrary polarization state expressed as follows

$$
G(r, \theta)=A(r)\left[\cos (\theta) \hat{e}_{L}+\sin (\theta) \hat{e}_{R}\right]
$$

is transformed, using a $q$-plate, into a vector mode in Eq. 4. The parameter $\theta$ can be tuned using a quarter-wave plate.

The projections onto the eigenstates of the Pauli operators are realised as in Fig. 1(a). Using a polarization grating based on geometric phase, the left- and right-handed circular polarization states are spatially separated and sent to two spatial modulators (SLMs), where the projections onto the eigenstates of the Pauli operators are performed with digital filters ${ }^{16-18}$. The projection measurements for scalar and vector modes, shown in Fig 1(a) and (b) respectively, are summarized symbolically in Table 1, where $\alpha$ is the intermodal phase in the superposition $\exp (i \ell \phi)+\exp (i \alpha) \exp (-i \ell \phi)$, and is given by $\alpha=0, \pi / 2 \ell, \pi / \ell, 3 \pi / 2 \ell$.

Table 1. Symbolic representation of the projective measurements onto eigenstates of the Pauli operators.

\begin{tabular}{ccccccc}
\hline Eigenstates & $\ell=1$ & $\ell=-1$ & $\alpha=0$ & $\alpha=\pi / 2$ & $\alpha=\pi$ & $\alpha=3 \pi / 2$ \\
\hline Left-circular & $I_{11}$ & $I_{12}$ & $I_{13}$ & $I_{14}$ & $I_{15}$ & $I_{16}$ \\
\hline Right-circular & $I_{21}$ & $I_{22}$ & $I_{23}$ & $I_{24}$ & $I_{25}$ & $I_{26}$ \\
\hline
\end{tabular}

The expectation values $\left\langle\sigma_{i}\right\rangle$ are computed as follows:

$$
\begin{aligned}
& \left\langle\sigma_{1}\right\rangle=\left(I_{13}+I_{23}\right)-\left(I_{15}+I_{25}\right) \\
& \left\langle\sigma_{2}\right\rangle=\left(I_{14}+I_{24}\right)-\left(I_{16}+I_{26}\right) \\
& \left\langle\sigma_{3}\right\rangle=\left(I_{11}+I_{21}\right)-\left(I_{12}+I_{22}\right)
\end{aligned}
$$

We varied the state of polarization before the $q$-plate from linear to circular (tuning the value of $\theta$ ), measured the expectation value of the Pauli operators, and obtained the plot in Fig. 2(a). We note that the variation of VQF measured is in agreement with the theory $(|\sin (2 \theta)|)$. The effect of tuning the parameter $\theta$ is shown in Fig. 2(b), where the intensity of the left- and right-circular components vary according to Eq. 1. 


\section{DISCUSSION AND CONCLUSION}

We have demonstrated a scheme to measure the degree of non-separability of a non-separable field. In the process, we introduced a quantitative measure of vectorness, derived from quantum tools that we call the vector quality factor. While we used vector vortex modes as an example, this scheme can in principle be adapted to arbitrary vector modes. Though the required projective measurements were performed by successively encoding holographic filters on a spatial light modulator, they can also be obtained by using demultiplexing holograms ${ }^{6}$.

\section{REFERENCES}

[1] Siegman, A., "Defining the effective radius of curvature for a nonideal optical beam," IEEE J. Quantum Electron. 27, 1146-1148 (1991).

[2] Siegman, A., "Laser beams and resonators: Beyond the 1960s," IEEE J. Quantum Electron. 6, 1389 - 1399 (2000).

[3] Zhan, Q., "Cylindrical vector beams: from mathematical concepts to applications," Adv. Opt. Phot. 1, 1-57 (2009).

[4] McLaren, M., Konrad, T., and Forbes, A., "Measuring the nonseparability of vector vortex beams," Phys. Rev. A 92, 023833 (2015).

[5] Ndagano, B., Brüning, R., McLaren, M., Duparré, M., and Forbes, A., "Fiber propagation of vector modes," Opt. Express 23, 17330-17336 (2015).

[6] Ndagano, B., Perez-Garcia, B., Roux, F. S., McLaren, M., Rosales-Guzmán, C., Zhang, Y., Mouane, O., Hernandez-Aranda, R. I., Konrad, T., and Forbes, A., "Process tomography of quantum channels using classical light," arXiv:1605.05144 (2016).

[7] Cox, M. A., Rosales-Guzmán, C., Lavery, M. P. J., Versfeld, D. J., and Forbes, A., "On the resilience of scalar and vector vortex modes in turbulence," Opt. Express 24, 18105-18113 (2016).

[8] Nape, I., Ndagano, B., Perez-Garcia, B., Scholes, S., Hernandez-Aranda, R. I., Konrad, T., and Forbes, A., "High-bit-rate quantum key distribution with entangled internal degrees of freedom of photons," arXiv:1605.05144 (2016).

[9] Cardano, F., Karimi, E., Slussarenko, S., Marrucci, L., de Lisio, C., and Santamato, E., "Polarization pattern of vector vortex beams generated by $q$-plates with different topological charges," Appl. Opt. 51, C1-C6 (2012).

[10] Chen, S., Zhou, X., Liu, Y., Ling, X., Luo, H., and Wen, S., "Generation of arbitrary cylindrical vector beams on the higher order Poincaré sphere," Opt. Lett. 39, 5274-5276 (2014).

[11] D'Ambrosio, V., Baccari, F., Slussarenko, S., Marrucci, L., and Sciarrino, F., "Arbitrary, direct and deterministic manipulation of vector beams via electrically-tuned q-plates," Sci. Rep. 5, 7840 (2015).

[12] Radwell, N., Hawley, R. D., Götte, J. B., and Franke-Arnold, S., "Achromatic vector vortex beams from a glass cone," Nat. Commun. 7, 10564 (2016).

[13] Naidoo, D., Roux, F. S., Dudley, A., Litvin, I., Piccirillo, B., Marrucci, L., and Forbes, A., "Controlled generation of higher-order Poincaré sphere beams from a laser," Nat. Phot. 10, 327-332 (2016).

[14] Inavalli, V. and Viswanathan, N., "Switchable vector vortex beam generation using an optical fiber," Opt. Commun. 283, 861-864 (2010).

[15] Milione, G., Lavery, M. P. J., Huang, H., Ren, Y., Xie, G., Nguyen, T. A., Karimi, E., Marrucci, L., Nolan, D. A., Alfano, R. R., and Willner, A. E., " $4 \times 20$ Gbit/s mode division multiplexing over free space using vector modes and a $q$-plate mode (de)multiplexer," Opt. Lett. 40, 1980-1983 (2015).

[16] Flamm, D., Naidoo, D., Schulze, C., Forbes, A., and Duparré, M., "Mode analysis with a spatial light modulator as a correlation filter," Opt. Lett. 37, 2478-2480 (2012).

[17] C. Schulze, A. D., Flamm, D., Duparré, M., and Forbes, A., "Measurement of the orbital angular momentum density of light by modal decomposition," New J. Phys. 15, 073025 (2013).

[18] Forbes, A., Dudley, A., and McLaren, M., "Creation and detection of optical modes with spatial light modulators," Adv. Opt. Phot. 8, 200-227 (2016). 